\title{
Altered Mechanisms Underlying the Abnormal Glutamate Release in Amyotrophic Lateral Sclerosis at a Pre-Symptomatic Stage of the Disease.
}

Tiziana Bonifacino $^{1 *}$, Laura Musazzi ${ }^{2}$, Marco Milanese ${ }^{1}$, Mara Seguini ${ }^{2}$, Antonella Marte ${ }^{3}$, Elena Gallia $^{1}$, Luca Cattaneo ${ }^{1}$, Franco Onofri ${ }^{3}$, Maurizio Popoli ${ }^{2}$, Giambattista Bonanno ${ }^{1}$

${ }^{1}$ Dept. of Pharmacy, Unit of Pharmacology and Toxicology, and Center of Excellence for Biomedical Research, University of Genoa, Italy.

${ }^{2}$ Dept. of Pharmacological and Biomolecular Sciences and Center of Excellence on Neurodegenerative Diseases, University of Milan, Italy

${ }^{3}$ Dept. of Experimental Medicine, Unit of Human Physiology, University of Genoa, Italy

\section{Introduction}

Amyotrophic Lateral Sclerosis (ALS) is a fatal neurodegenerative disease, characterized by loss of upper and lower motor neurons (MNs). It is well known that glutamate (Glu)-mediated excitotoxicity plays a major role in motor neuron damage and death, among several other cellular and molecular alterations. It has demonstrated that both excessive neuronal Glu release and defective glial Glu uptake contribute to increment the extracellular Glu levels and excitotoxicity.

\section{Aim}

We have investigated the release of Glu and the underlying mechanisms in spinal cord synaptosomes of SOD1 ${ }^{\text {G93A }}$ mice at a pre-symptomatic disease stage (30 days of life), in order to verify if the abnormal release occurs at this stage and to disclose the underlying mechanisms.

\section{Methods}

SOD $1^{\mathrm{G} 93 \mathrm{~A}}$ mice expressing high copy number of mutant human SOD1 carrying the G93A point mutation, the most popular animal model, and age-matched wtSOD1 control mice are used.

Synaptosomes were purified from SOD1 and SOD $1^{\mathrm{G} 93 \mathrm{~A}}$ spinal cord by homogenization and separation on discontinuous Percoll $\circledast$ gradients. Glu release was studied in superfusion. Ca2+ concentration was measured by fluorometric analysis. Western blots were performed according to Laemmli et al. (1970).

\section{Results}

We found that both basal and high $\mathrm{KCl}$ - or ionomycin-evoked Glu release were more elevated in the spinal cord of pre-symptomatic SOD $1^{\text {G93A }}$ respect to control mice. The surplus of Glu release was exocytotic in nature, supported by an increase of the readily releasable pool of vesicles (RRP). As to the mechanisms sustaining the abnormal Glu release, we found elevated cytosolic $\mathrm{Ca}^{2+}$ levels and increased phosphorylation of synapsin-I, that was causally related to the abnormal Glu release, being the latter normalized by entrapping synaptosomes with specific antibodies for synapsin-I phosphorylation sites. We also found increased 
phosphorylation of glycogen synthase kinase- 3 at the inhibitory sites, an event that favours SNARE protein assembly, increased number of SNARE complexes at the nerve terminal membrane, with no changes of SNARE protein expression, and increased expression of synaptotagmin-1 and $\beta$-Actin, out of an array of release-related presynaptic proteins whose expressions were unmodified.

\section{Conclusion}

Abnormal exocytotic Glu release occurs in the spinal cord of pre-symptomatic SOD $1^{\mathrm{G} 93 \mathrm{~A}}$ mice, similarly to what previously observed in late symptomatic SOD $1^{\mathrm{G} 93 \mathrm{~A}}$ mice. It mainly bases on an increased size of the RRP and on release facilitation; two events supported by plastic changes of specific pre-synaptic protein expression and function. Our results suggest that similar mechanisms can support the abnormal Glu release at pre- and late symptomatic stages of the disease. The precociousness of this phenomenon may imply that it represents a cause of the disease rather than a consequence of the neuronal damage during disease progression.

\section{Keywords}

amyotrophic lateral sclerosis, excessive Glu release, pre-synaptic protein alterations 